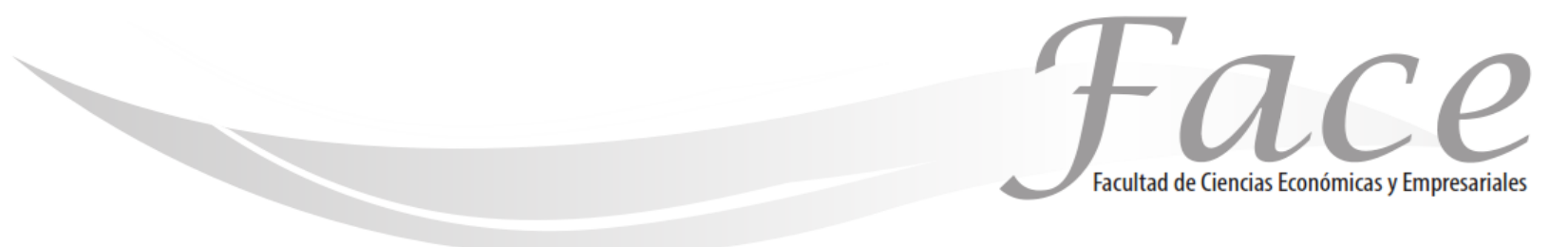

ISSN Impreso: 1794-9920

ISSN Electrónico: 2500-9338

Volumen $19-\mathrm{N}^{\circ} 1$

Año 2019

Págs. $27-38$

(c) (1) @)

\title{
CAPACIDADES DINÁMICAS DE MERCADOTECNIA, MERCADOTECNIA DIGITAL Y DESEMPEÑO EN LAS MIPYMES MEXICANAS
}

\author{
Catalina Gutiérrez Leefmans * \\ ORCID: https://orcid.org/0000-0001-9588-6753 \\ Rosa María Nava-Rogel** \\ ORCID: https://orcid.org/0000-0003-2611-3903 \\ Felipe Carlos Viesca-González *** \\ ORCID: https://orcid.org/0000-0003-0980-3507
}

Fecha de Recepción: Febrero 19 de 2019

Fecha de Aprobación: Abril 25 de 2019

\section{Resumen:}

Las capacidades dinámicas de mercadotecnia y la mercadotecnia digital influyen de manera positiva en el desempeño. Esta investigación busca conocer la situación de las MIPYMES en estas variables con el fin de desarrollar estrategias de mercadotecnia digital. Se realiza una investigación cuantitativa en línea a 140 empresas de distintos sectores en la República Mexicana. Los resultados indican que las empresas nuevas son las que más utilizan las herramientas digitales y tienen mayores capacidades dinámicas de mercadotecnia. Hay poca innovación en nuevos mercados por parte de las empresas del interior de la república, por lo que se infiere que sus estrategias son más locales. Las empresas medianas son las que utilizan más herramientas de mercadotecnia digital. Las empresas entrevistadas desconocen o no utilizan diversas herramientas de mercadotecnia digital, en particular las relacionadas con el análisis de métricas.

Palabras clave: Capacidades Dinámicas, Mercadotecnia digital, Desempeño, México

* Dra. en Ciencias Económico-Administrativas (DCEA). Universidad Autónoma del Estado de México. Profesora e investigadora de la Facultad de Contaduría y Administración Contacto: cgluniv@gmail.com

** Dra. en Ciencias Económico-Administrativas (DCEA). Universidad Autónoma del Estado de México. Jefa del departamento de investigación de la Facultad de Contaduría y Administración Contacto: cgluniv@gmail.com

*** Dr. en Administración. Universidad Autónoma del Estado de México. Profesor e investigador de la Facultad de Turismo Contacto: carvigo_9@hotmail.com 


\title{
DYNAMIC CAPACITIES OF MARKETING, DIGITAL MARKETING AND PERFORMANCE IN MEXICAN MIPYMES.
}

\begin{abstract}
:
Dynamic marketing and digital marketing capabilities have a positive influence on performance. This research seeks to know the situation of MSMEs in these variables in order to develop digital marketing strategies. Quantitative research is conducted online to 140 companies from different sectors in the Mexican Republic. The results indicate that new companies are the ones that most use digital tools and have greater dynamic marketing capabilities. There is little innovation in new markets by companies inside the republic, so it is inferred that their strategies are more local. Medium-sized companies are the ones that use the most digital marketing tools. The companies interviewed do not know or do not use various digital marketing tools, in particular those related to the analysis of metrics.
\end{abstract}

Keywords: Dynamic Capabilities, Digital Marketing, Performance, Mexico

\section{CAPACIDADES DINÂMICAS DE MARKETING, MARKETING DIGITAL E DESEMPENHO EM MIPÍMETOS MEXICANOS.}

\section{Resumo:}

O marketing dinâmico e as capacidades de marketing digital influenciam positivamente o desempenho. Esta pesquisa busca conhecer a situação das MPMEs nessas variáveis, a fim de desenvolver estratégias de marketing digital. A pesquisa quantitativa é realizada on-line para 140 empresas de diferentes setores da República Mexicana. Os resultados indicam que as novas empresas são as que mais usam ferramentas digitais e possuem maior capacidade de marketing dinâmico. Há pouca inovação em novos mercados pelas empresas dentro da república, portanto infere-se que suas estratégias são mais locais. Empresas de médio porte são as que mais utilizam ferramentas de marketing digital. As empresas entrevistadas não conhecem ou não utilizam diversas ferramentas de marketing digital, em especial aquelas relacionadas à análise de métricas.

Palavras-chave:Capacidades Dinâmicas, Marketing Digital, Performance, México. 


\section{INTRODUCCIÓN:}

La mercadotecnia es parte fundamental de la estrategia de los negocios y ha evolucionado rápidamente en el uso de Internet y las TICs (De Swaan Arons, Van den Driest y Weed, 2014). Asimismo, la mercadotecnia digital (que se refiere al uso de las TICs en la práctica mercadológica) es clave para los negocios ya que permite acercarse a los clientes, entenderlos mejor, agregar valor a los productos, aumentar los canales de distribución e impulsar las ventas (Chaffey y Smith, 2013).Las MIPYMES deberían poder identificar a la mercadotecnia digital como parte de la tecnología que ayuda a definir sus estrategias de negocios, ya que el no involucrarse activamente en estas prácticas podría mantenerlas al margen y ponerlas en riesgo de desaparecer (Mazzarol, 2015). Sin embargo deben ajustar su estructura organizacional, las capacidades, los recursos, los incentivos a las aplicaciones tecnológicas (BarbaSánchez et al., 2007).

En México más de $95 \%$ de las empresas son micro y ocupan al $45 \%$ de la población. Las empresas pequeñas y medianas, abarcan solo el $4.3 \%$ del total de las unidades económicas del país; sin embargo generan casi el $30 \%$ de las fuentes de empleo (INEGI, 2009). En comparación con otros países de la OCDE y otros países emergentes, México ha tenido un bajo crecimiento atribuible a los bajos niveles de productividad, por lo que las empresas, en particular las pequeñas, tienen entre sus retos mejorar $e$ incrementar el desarrollo productivo, desarrollar habilidades específicas e innovar (OCDE, 2012).

Esto indica que hay una necesidad de mejorar el desempeño de las MIPYMES mexicanas a partir de las TICs, y que la mercadotecnia digital puede ser un factor de progreso para las mismas. Sin embargo, existe muy poca investigación científica al respecto. Es por ello que esta investigación tiene el objetivo de conocer la situación de las capacidades dinámicas de mercadotecnia, el uso de la mercadotecnia digital y el desempeño obtenido en MIPYMES mexicanas que utilizan herramientas digitales para que las empresas puedan aprovechar esta información para la toma de decisiones estratégicas.

\section{MARCO TEÓRICO:}

Trainor et al. (2011:162) conceptualizan la mercadotecnia digital como "la integración de tecnología complementaria, negocios y recursos humanos que combinados influyen positivamente en el desempeño de la empresa". Otros autores también han asociado positivamente a la mercadotecnia digital con un mejor desempeño y una ventaja competitiva para las empresas (Brodie et al., 2007; Stokes, 2013; Ryan, 2014; Chaffey y Smith, 2013).

Por otro lado, algunos estudios han relacionado el concepto de las capacidades dinámicas con la mercadotecnia (Fang y Zou, 2008; Morgan, Vorhies y Mason, 2009; Morgan, 2012; Day, 1994 y 2011). Sin embargo, estudios más recientes sobre capacidades dinámicas de mercadotecnia y capacidades de mercadotecnia, aunque no hacen una referencia hacia la mercadotecnia digital como tal, si incluyen el uso de las herramientas digitales (Day, 2011; Morgan, 2012; De Swaan Arons et al., 2014). De igual forma, algunos autores no diferencian a las capacidades dinámicas de mercadotecnia de las capacidades de mercadotecnia, pero si incluyen en ellas características de capacidades dinámicas (De Swaan Arons et al., 2014, Chaffey, 2015).

Wang y Ahmed (2007) identificaron tres capacidades dinámicas: absorción, adaptativa e innovación, que, aunque no se enfocan en la mercadotecnia, auxiliaron a clasificar de una forma más precisa los resultados de los estudios encontrados relacionados con los conceptos "capacidades dinámicas de mercadotecnia", "capacidades de mercadotecnia" y "mercadotecnia digital". De esta forma se logró describir las características de tres capacidades dinámicas de mercadotecnia vinculadas con las herramientas digitales que para este estudio se denominaron: aprendizaje, adaptación e innovación.

La capacidad de aprendizaje se consideró la habilidad de reconocer el valor de información externa e interna, asimilarla y aplicarla con fines comerciales, transformándola en conocimiento (Wang y Ahmed, 2007), de manera vigilante (Day, 2011) y continua (Morgan, 2012), para tener un enfoque realista de la empresa y su entorno (De Swaan Aarons et al., 2014) que permita predecir cambios en el mercado (Morgan, 2012) a través de una conexión entre colaboradores (De Swaan Aarons et al., 2014) y una comunicación integral con el consumidor (Chaffey, 2015).

La capacidad de adaptación se definió como la habilidad de identificar y capitalizar las oportunidades de mercado (Wheeler, 2002) con base en la información aprendida y el balance entre la estrategias de exploración y aprovechamiento (Wang y Ahmed, 2007) y la experimentación continua (Day, 2011) de tal forma que se puedan retener, eliminar y adquirir recursos acordes con el entorno (Morgan, 2012) a través de una estructura organizacional ágil y flexible que pueda escalar ((De Swaan Aarons et al., 2014) y del compromiso de la gerencia (Chaffey, 2015). 
ISSN: 1794-9920 Impreso / Electrónico 2500-9338

Enero - Julio de 2019 - Volumen 19 Número 2, Año 2019 Págs. 27 - 38

Finalmente la capacidad de innovación se consideró la habilidad de la empresa de desarrollar nuevos productos y mercados mediante la alineación de una orientación innovadora estratégica con comportamientos y procedimientos innovadores (Wang y Ahmed, 2007) y mercadotecnia abierta (Day, 2011) de tal forma que se puedan retener, eliminar, adquirir y mejorar las capacidades acordes con el entorno (Morgan, 2012) logrando una experiencia integral del consumidor (Chaffey, 2015).

La mercadotecnia digital se examinó a partir del uso estratégico de las herramientas digitales para la ejecución de la práctica mercadológica con el fin de mejorar y optimizar su rendimiento (Chaffey y Smith, 2013). Finalmente, dado que tanto las capacidades dinámicas de mercadotecnia (Morgan, Vorhies y Mason, 2009; Morgan, 2012; Day, 1994 y 2011) como la mercadotecnia digital (Brodie et al., 2007; Stokes, 2013; Ryan, 2014; Chaffey y Smith, 2013) se han asociado de manera positiva con el desempeño de las empresas, se consideraron indicadores financieros en la mercadotecnia (Ambler y Kokkinaki, 2000; Clark, 2000) los cuales se denominaron desempeño financiero. A su vez se tomaron en cuenta medidas no financieras como calidad percibida, satisfacción del cliente, entre otras (Clark y Ambles, 2001) que se han obtenido a partir del uso de herramientas digitales y para este estudio se denominaron desempeño de mercado.

\section{MÉTODO:}

La investigación fue de campo, cuantitativa, de tipo no experimental y transeccional. La aplicación del cuestionario se llevó a cabo a través de una encuesta en línea dirigida a MIPYMES mexicanas que utilizan herramientas digitales, y que estuvo ubicada en la liga www.cglanalisis.com la segunda mitad del 2016. A partir de las dimensiones operacionales se consultaron algunos instrumentos relacionados y se construyó el cuestionario, el cual se verificó su confiabilidad a partir del alfa de Cronbach.

Se tuvo acceso a la base de datos del Instituto Mexiquense del Emprendedor (IME) que abarca empresas micro y pequeñas en el Estado de México. Para incluir empresas medianas, se seleccionó la base del Sistema de Información Empresarial Mexicano (SIEM) de empresas medianas en el Estado de México. La tercera base de datos se elaboró a partir de la relación de empresas listadas por el Instituto PYME, la cual incluye empresas pequeñas y medianas de toda la República Mexicana. Finalmente, se construyó la base de datos de las empresas con sello de confianza en la República Mexicana otorgado por la Asociación de Internet en México, por ser empresas con uso de herramientas digitales.
Las bases de datos seleccionadas reflejaron una población de 1,823 empresas. Se envió la invitación al total de la población seleccionada y se obtuvieron 140 respuestas efectivas de micro, pequeñas y medianas empresas de la República Mexicana de diversos sectores.

\section{HALLAZGOS:}

Los resultados están en una escala de 1 a10 que fue la escala de Likert que se utilizó en el cuestionario de investigación. Para una mejor explicación se agruparon de acuerdo a los siguientes elementos de estudio: antigüedad, tamaño, sector y ubicación geográfica de la empresa.Para la antigüedad de la empresa, se agruparon las que tenían un año de fundadas y posteriormente por cada cinco años antigüedad, siguiendo la clasificación que realiza el INEGI (2015) en su estudio de esperanza de vida de las empresas. En cuanto al tamaño de la empresa, la clasificación se realizó en micro, pequeña o mediana de acuerdo a los lineamientos que el INEGI utiliza por número de empleados, considerando también si la empresa era de servicio, comercio o manufactura. Con respecto a los sectores, se agruparon el sector comercial mayorista y minorista y se denominó "comercial"; lo relacionado a comunicaciones y tecnología se integró en "comunicaciones"; el grupo "otros servicios" incluyó educación, fianzas y seguros, limpieza, hotel y restaurante, esparcimiento y entretenimiento, salud, financieros e inmobiliarios; "industria" abarcó todo tipo de industria y finalmente se dejó "otros" que es una opción que incluía el cuestionario de acuerdo a la clasificación de las empresas del INEGI. Para la ubicación geográfica, la agrupación se realizó por empresas de la Ciudad de México, del Estado de México y de otros estados de la República.

\begin{tabular}{|c|c|c|}
\hline & No. de empresas & \%empresas \\
\hline \multicolumn{3}{|l|}{ Antigüedad } \\
\hline De 0 a 1 año & 19 & $13.6 \%$ \\
\hline Más de 1 a 5 años & 61 & $43.6 \%$ \\
\hline Más de 5 a 10 años & 28 & $20.0 \%$ \\
\hline Más de 10 a 15 años & 15 & $21.0 \%$ \\
\hline Más de 15 a 20 años & 7 & $9.8 \%$ \\
\hline Más de 20 años & 10 & $14.0 \%$ \\
\hline \multicolumn{3}{|l|}{ Factor F } \\
\hline ANOVA & Sig. & \\
\hline \multicolumn{3}{|l|}{ Tamaño } \\
\hline Micro & 103 & $73.6 \%$ \\
\hline Pequeña & 31 & $22.1 \%$ \\
\hline Mediana & 6 & $4.3 \%$ \\
\hline ANOVA de un factor & Sig. & \\
\hline \multicolumn{3}{|l|}{ Sector } \\
\hline Agropecuario & 3 & $2.1 \%$ \\
\hline Comercial & 20 & $14.3 \%$ \\
\hline Comunicaciones & 17 & $12.1 \%$ \\
\hline Otros servicios & 34 & $47.6 \%$ \\
\hline Servicios profesionales & 22 & $30.8 \%$ \\
\hline \multicolumn{3}{|c|}{ 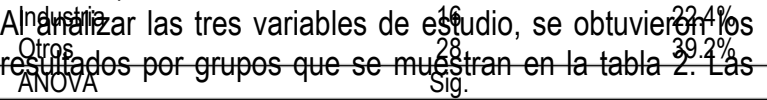 } \\
\hline \multicolumn{3}{|c|}{ Ubicación } \\
\hline Ciudad de México & 21 & $15.0 \%$ \\
\hline Estado de México & 88 & $62.9 \%$ \\
\hline Otros Estados & 31 & $22.1 \%$ \\
\hline ANOVA & Sig. & \\
\hline
\end{tabular}

Fuente: elaboración propia 


\section{CAPACIDADES DINÁMICAS DE MERCADOTECNIA, MERCADOTECNIA DIGITAL Y DESEMPEÑO \\ EN LAS MIPYMES MEXICANAS \\ Catalina Gutiérrez-Leefmans - Rosa María Nava-Rogel - Felipe Carlos Viesca-González}

empresas más jóvenes, menores a cinco años, son las que indicaron tener un mejor desempeño. Así mismo, son las empresas que obtuvieron el nivel más alto en capacidades dinámicas de mercadotecnia, en particular para la capacidad de aprendizaje, así como en uso de herramientas digitales.

Las diferencias entre los grupos por antigüedad de las empresas no fueron significativas para las variables de capacidades dinámicas de mercadotecnia y mercadotecnia digital, sin embargo si fueron significativas estas diferencias para la variable de desempeño, ya que obtuvo una significancia de 0.038 que es menor a .05 . antigüedad de la empresa ya que ubicó a todos los elementos en un mismo grupo.

Por otro lado, en lo que respecta al tamaño de las empresas, las medianas obtuvieron un desempeño más alto seguidas de las pequeñas y micro empresas. $Y$ aunque las medianas son las que utilizaron más herramientas de mercadotecnia digital, fueron las empresas pequeñas las que obtuvieron capacidades de mercadotecnia más altas, principalmente de aprendizaje. Sin embargo no existe diferencia significativa entre las tres clasificaciones por tamaño de la empresa.

Tabla N². Comparación de medias para las variables de estudio por grupos

\begin{tabular}{|c|c|c|c|}
\hline & $\begin{array}{l}\text { Capacidades } \\
\text { dinámicas de } \\
\text { mercadotecnia }\end{array}$ & Mercadotecnia digital & Desempeño \\
\hline \multicolumn{4}{|l|}{ Antiqüedad } \\
\hline De 0 a 1 año & 6.70 & 5.48 & 7.63 \\
\hline Más de 1 a 5 años & 6.25 & 5.34 & 6.71 \\
\hline Más de 5 a 10 años & 6.38 & 4.79 & 6.01 \\
\hline Más de 10 a 15 años & 6.18 & 4.59 & 5.77 \\
\hline Más de 15 a 20 años & 5.64 & 4.06 & 5.40 \\
\hline Más de 20 años & 6.18 & 4.59 & 5.90 \\
\hline Factor $\mathrm{F}$ & .498 & 1.102 & 2.430 \\
\hline ANOVA & .777 & .362 & .038 \\
\hline \multicolumn{4}{|l|}{ Tamaño } \\
\hline Micro & 6.21 & 4.87 & 6.38 \\
\hline Pequeña & 6.61 & 5.50 & 6.72 \\
\hline Mediana & 6.04 & 5.93 & 6.76 \\
\hline Factor F & .775 & 1.754 & .342 \\
\hline ANOVA de un factor & .463 & .177 & .711 \\
\hline \multicolumn{4}{|l|}{ Sector } \\
\hline Agropecuario & 5.47 & 2.52 & 4.18 \\
\hline Comercial & 5.99 & 4.49 & 6.18 \\
\hline Comunicaciones & 6.82 & 5.78 & 6.37 \\
\hline Otros servicios & 6.29 & 4.70 & 6.67 \\
\hline Servicios profesionales & 6.63 & 5.22 & 6.60 \\
\hline Industria & 6.00 & 5.33 & 6.80 \\
\hline Otros & 6.18 & 5.01 & 6.38 \\
\hline Factor $\mathrm{F}$ & .785 & 1.640 & .805 \\
\hline ANOVA & .583 & .141 & .567 \\
\hline \multicolumn{4}{|l|}{ Ubicación } \\
\hline Ciudad de México & 6.44 & 5.61 & 6.49 \\
\hline Estado de México & 6.31 & 4.98 & 6.63 \\
\hline Otros Estados & 6.15 & 4.91 & 6.01 \\
\hline Factor F & .202 & .912 & .949 \\
\hline ANOVA & .817 & .404 & .390 \\
\hline TOTAL Media & 6.29 & 5.05 & 6.47 \\
\hline
\end{tabular}

Fuente: elaboración propia

Para este valor se realizó la prueba de Tukey. Los resultados no confirmaron una diferencia significativa por 
ISSN: 1794-9920 Impreso / Electrónico 2500-9338

Enero - Julio de 2019 - Volumen 19 Número 2, Año 2019 Págs. 27 - 38

En cuanto a los resultados por sector, tampoco se observó una diferencia significativa aunque si es de notar que en el caso del sector agropecuario se obtuvieron los niveles más bajos de uso de mercadotecnia digital, desempeño y capacidades dinámicas de mercadotecnia. Los sectores relacionados a comunicación y tecnología, son los que obtuvieron los puntajes más altos en capacidades dinámicas de mercadotecnia y uso de mercadotecnia digital. Se destaca también el sector de servicios profesionales, otros servicios, así como industria, con los niveles más altos en desempeño.

La tabla 2 indica también que no hay una diferencia significativa entre los estados, aunque si se observa un mayor uso de herramientas de mercadotecnia digital en la Ciudad de México.
De manera general las medias de las tres variables fueron de 6.29 para las capacidades dinámicas de mercadotecnia, 5.05 para mercadotecnia digital y 6.47 para el desempeño, quedando los resultados con desviaciones estándar sin muestra de sesgos.

\section{Análisis de las capacidades dinámicas de mercadotecnia}

La tabla 3 muestra las medias por grupos de las tres capacidades dinámicas de mercadotecnia. En esta clasificación por grupos no se revelaron diferencias significativas en ninguno de los casos, aunque destacan nuevamente las empresas menores a un año de antigüedad con las mayores capacidades de los tres tipos. De igual forma, los sectores de comunicaciones y servicios profesionales fueron los que tienen en promedio las capacidades más altas.

Tabla $\mathrm{N}^{\circ} 3$

Comparación de Medias Para Capacidades Dinámicas de Mercadotecnia por Grupos

\begin{tabular}{|c|c|c|c|}
\hline & Aprendizaje & Adaptación & Innovación \\
\hline \multicolumn{4}{|l|}{ Antigüedad } \\
\hline De 0 a 1 año & 7.27 & 6.43 & 6.40 \\
\hline Más de 1 a 5 años & 6.76 & 5.92 & 6.06 \\
\hline Más de 5 a 10 años & 6.90 & 6.40 & 5.84 \\
\hline Más de 10 a 15 años & 6.41 & 6.29 & 5.85 \\
\hline Más de 15 a 20 años & 6.32 & 5.48 & 5.12 \\
\hline Más de 20 años & 6.81 & 5.67 & 6.05 \\
\hline Factor $\mathrm{F}$ & .653 & .657 & .572 \\
\hline ANOVA & .660 & .657 & .721 \\
\hline \multicolumn{4}{|l|}{ Tamaño } \\
\hline Micro & 6.73 & 5.97 & 5.94 \\
\hline Pequeña & 7.04 & 6.52 & 6.27 \\
\hline Mediana & 6.78 & 5.87 & 5.47 \\
\hline Factor $\mathrm{F}$ & .452 & 1.084 & .623 \\
\hline ANOVA de un factor & .637 & .341 & .538 \\
\hline \multicolumn{4}{|l|}{ Sector } \\
\hline Agropecuario & 5.97 & 5.30 & 5.13 \\
\hline Comercial & 6.77 & 5.70 & 5.50 \\
\hline Comunicaciones & 7.25 & 6.65 & 6.58 \\
\hline Otros servicios & 6.79 & 6.18 & 5.92 \\
\hline Servicios profesionales & 7.09 & 6.56 & 6.23 \\
\hline Industria & 6.68 & 5.40 & 5.93 \\
\hline Otros & 6.51 & 6.01 & 6.03 \\
\hline Factor $\mathrm{F}$ & .635 & 1.093 & .702 \\
\hline ANOVA & .702 & .370 & .648 \\
\hline \multicolumn{4}{|l|}{ UBICACIÓN } \\
\hline Ciudad de México & 6.95 & 6.18 & 6.18 \\
\hline Estado de México & 6.81 & 6.10 & 6.02 \\
\hline Otros Estados & 6.69 & 5.97 & 5.80 \\
\hline Factor F & .174 & .091 & .289 \\
\hline ANOVA & .841 & .913 & .749 \\
\hline TOTAL Media & 6.80 & 6.08 & 5.99 \\
\hline
\end{tabular}

Fuente: elaboración propia 


\section{CAPACIDADES DINÁMICAS DE MERCADOTECNIA, MERCADOTECNIA DIGITAL Y DESEMPEÑO \\ EN LAS MIPYMES MEXICANAS \\ Catalina Gutiérrez-Leefmans - Rosa María Nava-Rogel - Felipe Carlos Viesca-González}

Se observa también que del valor de las medias totales por capacidad dinámica de mercadotecnia, las empresas obtuvieron más capacidad de aprendizaje que de adaptación e innovación, quedando esta última con la media más baja.

El análisis de los resultados de estas tres capacidades, permite inferir que las empresas han logrado desarrollar más las capacidades relacionadas con factores internos que con los externos. Es decir, conocen más sus recursos internos que la situación del mercado, realizan adaptación interna más que buscar la conexión con terceros y adaptación externa.

Varios autores mencionan la necesidad de obtener y usar información y conocimiento externos valiosos para desarrollar actividades de mercadotecnia digital (Wu et al. 2003; Brodie et al. 2007, Trainor et al. 2011; Morgan et al. 2009), esto indica que hay un área de oportunidad en las empresas de desarrollar sus capacidades mas hacia lo relacionado al entorno y sobre todo de tener una actividades estratégicas que vayan más de afuera hacia adentro que de adentro hacia fuera como lo indica Day (2011).
Por otro lado, la innovación se da en productos y servicios más que en el desarrollo de nuevos mercados, y la Ciudad de México es la que más innova en mercados a comparación del resto de la República. Eso puede significar que las MIPYMES del interior de la república tienen una visión más local que las limita en sus posibilidades de expansión y crecimiento.

\section{Análisis de la mercadotecnia digital}

Para identificar el nivel de uso de la mercadotecnia digital se clasificó el promedio de la variable mercadotecnia digital en cinco niveles de acuerdo a los resultados de la escala de Likert utilizada en el cuestionario. Es así que los que obtuvieron 102 puntos se les asignó nivel Muy Bajo, valores de 3 y 4 nivel Bajo, 5 y 6 nivel Medio, 7 y 8 nivel Alto y 9 y 10 nivel Muy Alto.

Al analizar el nivel de uso de herramientas de mercadotecnia digital en la tabla 4, se identificó una fuerte relación positiva con las capacidades dinámicas de mercadotecnia, es decir, a mayor uso de herramientas digitales, mayores capacidades dinámicas de mercadotecnia. De igual forma a mayor uso de las herramientas digitales, hubo mayor desempeño de las empresas. La diferencia entre los niveles de uso de la mercadotecnia digital tanto para las capacidades dinámicas de mercadotecnia como para el desempeño es significativa como lo indica la prueba de ANOVA.

Tabla $\mathrm{N}^{\circ} 4$.

Capacidades Dinámicas de Mercadotecnia Y Desempeño

por Nivel de Uso de Herramientas De Mercadotecnia Digital

\begin{tabular}{lccccccc}
\hline USO de MD & $\begin{array}{c}\text { No. de } \\
\text { empresas }\end{array}$ & $\begin{array}{c}\% \\
\text { empresas }\end{array}$ & Aprendizaje & Adaptación & Innovación & CDM & Desempeño \\
\hline Muy bajo & 24 & $17.1 \%$ & 5.34 & 4.41 & 4.23 & 4.66 & 4.06 \\
Bajo & 48 & $34.3 \%$ & 6.38 & 5.43 & 5.67 & 5.83 & 5.78 \\
Medio & 42 & $30.0 \%$ & 7.36 & 6.67 & 6.39 & 6.81 & 7.51 \\
Alto & 23 & $32.2 \%$ & 7.94 & 7.74 & 7.51 & 7.73 & 8.18 \\
Muy alto & 3 & $4.2 \%$ & 8.74 & 9.14 & 8.09 & 8.66 & 9.21 \\
ANOVA & Sig. & & .000 & .000 & .000 & .000 & .000 \\
\hline
\end{tabular}

Fuente: elaboración propia

Lo anterior no solo evidencia la relación entre la mercadotecnia digital y las variables de capacidades de mercadotecnia y desempeño, sino que también explica que 
esta relación es positiva y significativa. Las pruebas de Tukey para cada una de las capacidades dinámicas de mercadotecnia, el promedio de las capacidades, así como el desempeño, revelan que efectivamente existen diferencias significativas al agrupar la mercadotecnia digital por niveles.

Estos resultados contribuyen a afirmar la relación positiva entre la mercadotecnia digital y el desempeño como una ventaja competitiva para las empresas (Brodie et al., 2007; Trainor et al., 2011; Stokes, 2013; Chaffey y Smith, 2013; Ryan, 2014).
Esto indica que las empresas no conocen realmente las posibilidades de la mercadotecnia digital ni todas las herramientas que se pueden utilizar para la mercadotecnia. Esto coincide con lo que mencionan De Swaan Aarons et al. (2014) al respecto de que las empresas no han evolucionado al igual que los avances tecnológicos de la mercadotecnia.

Figura $\mathrm{N}^{\circ} 1$.

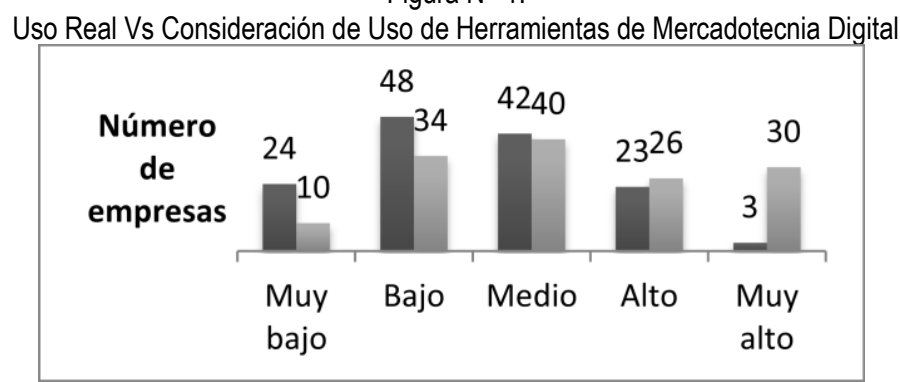

Fuente: elaboración propia a partir de los resultados

Otro resultado que se manifestó de forma evidente, fue que las empresas consideran que utilizan la mercadotecnia digital en un nivel superior a lo que en realidad la utilizan en comparación con otras empresas. Una de las preguntas iniciales de filtro del cuestionario solicitaba que se indicara si el nivel de uso de la mercadotecnia digital por parte de la empresa era Muy Alto, Alto, Medio, Bajo y Muy Bajo. Si se comparan los resultados de esta respuesta con los del uso real de la mercadotecnia digital dado por el promedio de los resultados de las preguntas relacionadas de esta variable, se obtiene una diferencia importante entre lo que consideran las empresas y la realidad.

La figura 1 permite apreciar que las empresas consideran que tienen un nivel alto de uso de herramientas de mercadotecnia digital, auto-clasificándose muchas de ellas en los niveles de uso Muy alto y Alto, siendo que más bien la mayoría tiene un nivel bajo en el uso de estas herramientas.
En cuanto al uso de las herramientas de mercadotecnia digital, los resultados indicaron que las herramientas relacionadas con las comunicaciones con el cliente son las que más utilizaban las empresas. Cabe destacar que la pregunta relacionada al correo electrónico fue la herramienta que obtuvo el puntaje más alto de uso, mientras que la herramienta que menos se utilizó fue la de publicidad pagada en banners.

El factor de base de datos fue el más utilizado por las empresas y el menos utilizado el de publicidad. Las preguntas agrupadas en el factor de base de datos se refieren al uso de correo electrónico y el sistema de registro de los clientes. El correo electrónico es una herramienta de uso básico y con varios años de antigüedad lo que explica este resultado. Las preguntas relacionadas con el factor publicidad se refieren a la publicidad pagada en redes sociales y en banners, y el haber obtenido los niveles más bajos da a entender que las MIPYMES en México invierten poco en publicidad en línea todavía.

Las herramientas relacionadas a analíticos y servicio al cliente se encuentran subutilizadas, al contar con niveles de uso de la media de la escala o menos. De aquí se infiere que el bajo uso de herramientas estratégicas como el análisis de métricas pudiera darse por su complejidad para los gerentes de las empresas. 


\section{CAPACIDADES DINÁMICAS DE MERCADOTECNIA, MERCADOTECNIA DIGITAL Y DESEMPEÑO EN LAS MIPYMES MEXICANAS \\ Catalina Gutiérrez-Leefmans - Rosa María Nava-Rogel - Felipe Carlos Viesca-González}

Las preguntas del factor de servicio al cliente se relacionaron con el uso de herramientas como el chat y las apps para dar servicio al cliente, así como el uso del sitio web para dar noticias. El factor de analíticos agrupó las preguntas relacionadas al uso de herramientas de comparación y análisis de métricas como Alexa, Google Analytics, Google Adwords, pruebas A/B y optimización en motores de búsqueda.

Entre las preguntas que se agruparon en el factor de comunicación se estuvieron las relacionadas a redes sociales y los medios para resolver dudas, hacer pedidos y dar seguimiento a clientes. El factor usabilidad incluyó las herramientas relacionadas al diseño del sitio web, su facilidad de navegación, verificación de su funcionamiento, administración del contenido y su adaptación en diseño para celulares, tabletas, etc.

Al analizar el uso de las herramientas de mercadotecnia digital de acuerdo a la antigüedad de la empresa se observó que las empresas más jóvenes utilizaban principalmente las herramientas de comunicación, existiendo una diferencia significativa entre los grupos por años de antigüedad. Entre más antigua la empresa había un menor uso de las herramientas digitales, destacando que lo que menos utilizaban era la publicidad y en el caso de empresas de 15 a 20 años de antigüedad, había un bajo uso de herramientas de servicio al cliente. Esto coincide con Saavedra y Tapia (2013) que señalan que las empresas nuevas nacen utilizando la tecnología.

Con respecto al tamaño de la empresa, se observó claramente que entre más grande es la empresa, más herramientas digitales utilizaban, destacándose las herramientas de usabilidad y base de datos cuyas ANOVAS fueron significativas. Esto indica que las empresas medianas, a diferencia de las mas pequeñas son más estratégicas al procurar generar información relacionada al cliente y en el desarrollo de su sitio web. Se infiere que al haber más gente involucrada en la empresa se pueden realizar mas actividades de mercadotecnia digital, además de las de comunicación.

Al analizar el comportamiento del uso de las herramientas por sector, base de datos fue el factor que presentó mayor uso. La diferencia entre los sectores para la mayoría de los factores de mercadotecnia digital no mostraron una diferencia significativa, sin embargo, el factor de servicio al cliente si la reflejó, siendo el de comunicaciones el que mas utilizó estas herramientas y el sector agropecuario el que menos.

En cuanto a la ubicación geográfica de las empresas, se observó un mayor uso de éstas herramientas digitales en la Ciudad de México, seguida por el Estado de México y después otros estados de la Republica. Las herramientas que más se utilizaron en la Ciudad de México son las de base de datos y usabilidad. En toda la República se observó un uso medio de herramientas de comunicación, mientras que en el Estado de México hubo un bajo uso de herramientas de analíticos, y en otros estados, un bajo uso de herramientas de servicio al cliente. Aunque las diferencias no fueron significativas.

Los resultados anteriores permiten confirmar que las herramientas más utilizadas son las de base de datos, comunicación y usabilidad y que se muestra un bajo uso de las herramientas de servicio al cliente, publicidad y analíticos. Dado que la usabilidad se refiere al diseño de la página web y base de datos al registro de los usuarios 0 clientes, se deduce entonces que los esfuerzos de las empresas se están enfocando en la imagen y la comunicación con el cliente, pero hay poco conocimiento de la parte estratégica de la mercadotecnia digital al desconocer las herramientas que contribuyen al análisis de la información para la toma de decisiones y la mejora del servicio al cliente, así como poca inversión en publicidad digital. Esto coincide con Demuner et al. (2104) al afirmar que las empresas en México se inclinan más por herramientas básicas de tecnología y el rezago de las MIPYMES aumenta de manera importante cuando se consideran las tecnologías de nivel más complejo y sofisticado.

Así mismo, se evidencia que no hay diferencias significativas en el estudio de la mercadotecnia digital entre grupos por tamaño, ubicación, sector o antigüedad de la empresa. Lo cual indica que hay poca relevancia en realizar esta diferenciación y fortalece el que ésta investigación se haya realizado sin estar dirigida a un sector en particular.

\section{Análisis del desempeño}

Los resultados indicaron que las empresas más jóvenes (menores a un año) obtuvieron un mejor desempeño a partir del uso de las herramientas de mercadotecnia digital, sobre todo en lo que se refiere al desempeño de mercado. En cuanto a tamaño de la empresa no se presentaron diferencias significativas entre la micro, pequeña y mediana empresa.

Los resultados de desempeño por sector mostraron nuevamente un mayor desempeño de mercado que financiero y al igual que en las otras dimensiones, los sectores de comunicaciones y servicios profesionales obtuvieron los valores más altos, mientras que el sector agropecuario obtuvo los resultados más bajos, aunque las diferencias no fueron significativas.

Para la agrupación por ubicación, el desempeño de mercado obtuvo resultados mayores al desempeño financiero sin embargo, la diferencia entre estados de la república, no fue significativa.

De manera general estos datos indican que a partir del uso de las herramientas digitales en la mercadotecnia, las empresas logran un desempeño mayor en aspectos de mercado que en financieros. Esto pudiera significar que las 
ISSN: 1794-9920 Impreso / Electrónico 2500-9338

Enero - Julio de 2019 - Volumen 19 Número 2, Año 2019 Págs. 27 - 38

empresas no miden los resultados financieros, sin embargo a partir de la propia experiencia, conocen al cliente y a la competencia.

\section{CONCLUSIONES:}

El análisis permitió identificar a las empresas más jóvenes como aquellas con mayores capacidades dinámicas de mercadotecnia, mayor uso de herramientas de mercadotecnia digital y mayor desempeño. Dado que se ha evidenciado que a mayor uso de las herramientas digitales hay un mayor desempeño, se apoya el argumento de que las empresas nuevas nacen utilizando las tecnologías. En la actualidad existen diversas herramientas digitales y aplicaciones diseñadas especialmente para los emprendedores. Los nuevos empresarios saben que deben apoyarse en las nuevas tecnologías para ser más exitosas (Saavedra y Tapia, 2013). Las MIPYMES mexicanas que llevan más años deben trabajar en incorporar en su estrategia el uso de herramientas digitales para la mercadotecnia.

Por otro lado, las clasificaciones por antigüedad, ubicación, sector y tamaño de la empresa no representaron diferencias significativas entre los grupos, al menos en la muestra estudiada. Sin embargo, si resaltaron los sectores de comunicación y servicios como lo sectores con los niveles más altos en las tres variables de estudio, mientras que el sector agropecuario fue el de resultados más bajos.

Así mismo, las empresas más grandes, en este caso, las medianas, fueron las que más utilizaban las herramientas de mercadotecnia digital, pero al no ser significativas las diferencias, se requiere hacer una comparación con las empresas grandes, que son las que adoptan las innovaciones con mayor rapidez (Hall y Khan, 2003).

En cuanto a las capacidades dinámicas de mercadotecnia, la capacidad de aprendizaje y de adaptación dejaron ver que hay un mayor enfoque en los factores internos que los externos, lo cual es una debilidad para las empresas ya que Day (2011) menciona que las nuevas capacidades adaptativas deben tener un enfoque de afuera hacia adentro. Así mismo, Barba-Sánchez et al., 2007 indican que las MIPYMES deben ajustar su estructura organizacional, las capacidades, los recursos, los incentivos a las aplicaciones tecnológicas que brinda el entorno.

La Ciudad de México obtuvo los resultados más altos y de manera significativa en cuanto a la innovación de mercados. De aquí se pudiera inferir que el resto de los estados de la república tienen una visión más local que la Ciudad de México. Aunque esta inferencia deberá corroborarse, el resultado implica un área de oportunidad para las MIPYMES de la república mexicana.

El contraste entre la percepción de uso de herramientas digitales vs el uso real de las herramientas indica que muchas empresas no conocen las posibilidades de la mercadotecnia digital. Al responder que tienen un nivel alto de uso de mercadotecnia digital, y posteriormente responder que no utilizan las herramientas, indica que falta instrucción sobre la mercadotecnia digital.

Este resultado coincide con De Swaan Arons et al. (2014) en que las empresas no han evolucionado de la misma forma que la mercadotecnia digital y deben identificar las capacidades que llevan a una excelencia mercadológica, así como las estructuras y formas de trabajo que las apoyan. De igual forma Stone (2014), indica que el entrenamiento, la comunicación interna y las estructuras administrativas deben dirigirse en la integración como requisito de supervivencia, y los directivos deben estar conscientes de ello.

Es así que se concluye que la mercadotecnia digital se relaciona de manera positiva con las capacidades dinámicas de mercadotecnia y el desempeño de MIPYMES mexicanas que utilizan herramientas digitales, de tal modo que el desarrollo de éstas capacidades en sus empresas apoyadas por el uso estratégico de mercadotecnia digital puede potenciar su desempeño.

\section{REFERENCIAS:}

Ambler, T., \& Kokkinaki, F. (2000). Marketing performance measurement: which way is up?. International Journal of Business Performance Management, 2(1-3), 72-85.

Barba-Sánchez, V., Martínez-Ruiz, M. D. P., \& JiménezZarco, A. I. (2007). Drivers, benefits and challenges of ICT adoption by small and medium sized enterprises (SMEs): a literature review. Problems and Perspectives in Management, 5(1), 104-115.

Brodie, R. J., Winklhofer, H., Coviello, N. E., \& Johnston, W. J. (2007). Is e-marketing coming of age? An examination of the penetration of e-marketing and firm performance. Journal of Interactive Marketing, 21(1): 2-21. 


\section{CAPACIDADES DINÁMICAS DE MERCADOTECNIA, MERCADOTECNIA DIGITAL Y DESEMPEÑO \\ EN LAS MIPYMES MEXICANAS \\ Catalina Gutiérrez-Leefmans - Rosa María Nava-Rogel - Felipe Carlos Viesca-González}

Chaffey, D. (2015). Digital business and E-commerce management. Pearson Education Limited.

Chaffey, D., \& Smith, P. R. (2013). E-marketing Excellence: Planning and Optimizing your Digital Marketing. Routledge.

Clark, B. H. (2000). Managerial perceptions of marketing performance: efficiency, adaptability, effectiveness and satisfaction. Journal of Strategic Marketing, 8(1), 3-25.

Clark, B. H., \& Ambler, T. (2001). Marketing performance measurement: evolution of research and practice. International journal of business performance management, 3(2-4), 231-244.

Day, G. S. (1994). The capabilities of market-driven organizations. the Journal of Marketing, 37-52.

Day, G. S. (2011). Closing the marketing capabilities gap. Journal of Marketing, 75(4): 183-195.

De Swaan Arons, M., Van Den Driest, F., y Weed, K. (2014). The ultimate marketing machine. Harvard Business Review, 92(7), 54-63.

Demuner, M. D. R., Nava, R. M., y Gómez M. D. R. (Noviembre, 2014). Las tecnologías de información y comunicación en las pequeñas y medianas empresas. Congreso Virtual sobre Tecnología, Educación y Sociedad (Vol. 1, No. 2).

Fang, E. E., \& Zou, S. (2009). Antecedents and consequences of marketing dynamic capabilities in international joint ventures. Journal of International Business Studies, 40(5), 742-761.

Hall, B. H., \& Khan, B. (2003). Adoption of new technology (No. w9730). National Bureau of Economic Research.

INEGI (2009). Micro, pequeña, mediana y gran empresa: estratificación de los establecimientos. Censos Económicos, Instituto Nacional de Estadística y Geografía, México.

INEGI (2015) Esperanza de vida de las empresas. Instituto Nacional de Estadística y Geografía, México. Recuperado de http://www.beta.inegi.org.mx/temas/evnm/
Mazzarol, T. (2015). SMEs engagement with e-commerce, e-business and e-marketing. Small Enterprise Research, (ahead-of-print), 1-12.

Morgan, N. A. (2012). Marketing and business performance. Journal of the Academy of Marketing Science, 40(1), 102-119.

Morgan, N. A., Vorhies, D. W., \& Mason, C. H. (2009). Market orientation, marketing capabilities, and firm performance. Strategic Management Journal, 30(8), 909-920.

OCDE (2012) Internet Economy Outlook 2012 | OECD READ edition. (s. f.). Recuperado 19 de septiembre de 2013, a partir de http://www.keepeek.com/oecd/media/scienceand-technology/oecd-internet-economy-outlook2012_9789264086463-en\#page151

Ryan, D. (2014). Understanding digital marketing: marketing strategies for engaging the digital generation. Kogan Page Publishers.

Saavedra García, M. L., \& Tapia Sánchez, B. (2013). El uso de las tecnologías de información y comunicación TIC en las micro, pequeñas y medianas empresas (MIPyME) industriales mexicanas. Enl@ ce: Revista Venezolana de Información, tecnología y conocimiento, 10(1).

Stokes, R. (2013). E-Marketing: The Essential Guide to Digital Marketing. Quirk Education: Cape Town, South Africa.

Stone, M. (2014). The new (and ever-evolving) direct and digital marketing ecosystem. Journal of Direct, Data and Digital Marketing Practice, 16(2), 71-74.

Trainor, K. J., Rapp, A., Beitelspacher, L. S., \& Schillewaert, N. (2011). Integrating information technology and marketing: An examination of the drivers and outcomes of e-Marketing capability. Industrial Marketing Management, 40(1), 162-174.

Wang, C. L., \& Ahmed, P. K. (2007). Dynamic capabilities: $A$ review and research agenda. International Journal of Management Reviews, 9(1): 31-51. 
ISSN: 1794-9920 Impreso / Electrónico 2500-9338

Enero - Julio de 2019 - Volumen 19 Número 2, Año 2019 Págs. 27 - 38

Wheeler, B. C. (2002). NEBIC: a dynamic capabilities theory for assessing net-enablement. Information Systems Research, 13(2), 125-146.

Wu, F., Mahajan, V., \& Balasubramanian, S. (2003). An analysis of e-business adoption and its impact on business performance. Journal of the Academy of Marketing Science, 31(4), 425-447. 